\title{
Теория формальных языков и тождества неассоциативных алгебр
}

\author{
Зайцев Михаил Владимирович, Реповш Душан Душанович
}

В статве рассматриваются числовые характеристики тождеств неассоциативных алгебр. Предложен метод построения по двоичному слову $w$ алгебры $A(w) c$ заданными свойствами функции роста коразмерностей. При этом рост коразмерностей алгебры $A(w)$ полностъю определяется комбинаторной сложностъю языка подслов слова $w$.

В работе используются результаты теории формальнных языков для решения целого ряда проблем PI-теории, т.е. теории тождественных соотношений в алгебрах. Комбинаторные свойства языков, состоящих из подслов бесконечного слова, неоднократно использовались для построения различных примеров асимптотического поведения количественных характеристик, связанных с тождествами (см., например, $[4,5])$. Альтернативная конструкция построения алгебры по бесконечному двоичному слову была предложена в работе [11]. Мы модернизируем подход работы [11], что позволяет нам связать числовые инварианты тождеств построенной алгебры с комбинаторной сложностью языка, задаваемого изначальным словом.

Сначала напомним необходимые понятия из РІ-теории. Пусть $\Phi-$ поле нулевой характеристики и $A-$ некоторая (не обязательно ассоциативная) алгебра над $\Phi$. Обозначим через $\Phi\{X\}$ абсолютно свободную алгебру над $\Phi$ с бесконечным множеством порождающих $X$. Тогда совокупность всех тождеств алгебры $A$ образует двусторонний идеал $\operatorname{Id}(\mathrm{A})$ в $\Phi\{X\}$. Хорошо известно, что идеал $\mathrm{Id}(\mathrm{A})$ полностью определяется своими полилинейными компонентами, т.е. совокупностью подпространств $\operatorname{Id}(\mathrm{A}) \cap \mathrm{P}_{\mathrm{n}}, \mathrm{n}=1,2, \ldots$, где $P_{n}$ - подпространство полилинейных полиномов от $x_{1}, \ldots, x_{n}$ в $\Phi\{X\}$. Все необходимые определения и понятия из PI-теории можно найти в [8]. Обозначим

$$
P_{n}(A)=\frac{P_{n}}{\operatorname{Id}(\mathrm{A}) \cap \mathrm{P}_{\mathrm{n}}}, \quad c_{n}(A)=\operatorname{dim} P_{n}(A) .
$$

Величина $c_{n}(A)$ называется $n$-й коразмерностью алгебры $A$. В работе [12] было установлено, что последовательность $\left\{c_{n}(A)\right\}$ экспоненциально ограничена для любой ассциативной PI-алгебры. В конце 80-х годов прошлого века Амицур выдвинул гипотезу, что последовательность $\sqrt[n]{c_{n}(A)}$ имеет предел, который является неотрицательным целым числом. Позже Регев выдвинул более сильное предположение, что $c_{n}(A) \sim C n^{t} d^{n}$, где $C$ - константа, соотношение $f(n) \sim g(n)$ означает, что $\lim _{n \rightarrow \infty}(f(n) / g(n))=1$. Более того, число $d$ должно быть целым, a $t$ - полуцелым. Гипотеза Регева означает, что сущесвуют три предела

$$
d=\lim _{n \rightarrow \infty} \sqrt[n]{c_{n}(A)}, t=\lim _{n \rightarrow \infty} \log _{n} \frac{c_{n}(A)}{d^{n}}, \quad C=\lim _{n \rightarrow \infty} \frac{c_{n}(A)}{n^{t} d^{n}},
$$


которые можно назвать 1-м, 2-м и 3-м приближениями соответственно. Число $\exp (A)=d$ при этом называется PI-экспонентой алгебры $A$. Отметим, что для ассоциативных алгебр гипотеза Регева в 1-м и 2-м приближениях подтвердилась $[2,6,7,9]$, а вопрос о третьем приближении до сих пор открыт. Более того, оказалось, что в случае $\exp (A)=1$ последовательность $\left\{c_{n}(A)\right\}$ полиномиально ограничена, т.е. не может иметь промежуточный рост.

В общем неассоциативном случае для любого вещественного $\alpha>1$ есть примеры алгебр, у которых $c_{n}(A)$ растет асимптотически как $\alpha^{n}$ [5]. Кроме того, есть примеры, когда последовательность $\left\{c_{n}(A)\right\}$ экспоненциально ограничена, но первого предела из (2) не существует [14]. Во втором приближении в случае отсутствия ассоциативности гипотеза Регева тоже не подтвердилась [10]. Есть и примеры алгебр с промежуточным ростом последовательности коразмерностей [4]. Но во всех этих примерах $c_{n}(A)$ асимптотически растет как $n^{n^{\beta}}$ с $0<\beta<1$.

Основная цель настоящей работы - показать, что класс функций промежуточного роста, реализуемых в виде последовательностей $\left\{c_{n}(A)\right\}$, гораздо шире и что в третьем приближении (при условии существования 1-го и 2-го пределов в (2) гипотеза Регева также неверна.

Перейдем к реализации основной конструкции, т.е. к построению алгебры по заданному бесконечному двоичному слову. Напомним, что формальным языком, задаваемым словом $w$, называется совокупность всех конечных подслов $w$, а комбинаторной сложностью этого языка и самого слова $w$ называется функция $\operatorname{Comp}_{w}: \mathbb{N} \rightarrow \mathbb{N}$, где $\operatorname{Comp}_{w}(n)$ - число различных подслов длины $n$ в $w$.

Пусть $w=w_{1} w_{2} \ldots$ - бесконечное слово в алфавите $\{0 ; 1\}$. Обозначим через $A(w)$ алгебру, порожденную двумя элементами $a$ и $b_{0}$, с базисом $\left\{a, b_{0}, b_{1}, \ldots\right\}$, умножение в котором задается следующим образом. Если $w_{1}=1$, то $b_{1}=a b_{0}$, если же $w_{1}=0$, то $b_{0} a=b_{1}$. Пусть $b_{1}, \ldots, b_{k-1}$ уже определены. Тогда

$$
b_{k}=\left\{\begin{array}{lll}
a b_{k-1}, & \text { если } & w_{k}=1, \\
b_{k-1} a, & \text { если } & w_{k}=0 .
\end{array}\right.
$$

Все остальные произведения базисных элементов равны нулю. При любом $w$ алгебра $A(w)$ удовлетворяет тождеству

$$
\left(x_{1} x_{2}\right)\left(x_{3} x_{4}\right) \equiv 0
$$

поэтому коразмерности можно считать не в $\Phi\{X\}$, а в свободной метабелевой алгебре $M\{X\}$, т.е. в относительно свободной алгебре многообразия, заданного тождеством (4).

Для элемента $x$ метабелевой алгебры обозначим через $R_{x}$ и $L_{x}$ операторы правого и левого умножения на $x$ соответственно. И тот и другой операторы будем писать справа, т.е. $y R_{x}=y x, y L_{x}=x y$. Для произвольного двоичного слова $u=u_{1} \ldots u_{m}$ и для $y, x_{1}, \ldots, x_{m} \in X \subset M\{X\}$ обозначим через $y u\left(x_{1}, \ldots, x_{m}\right)$ одночлен $y T_{1} \cdots T_{m}$, где $T_{i}=R_{x_{i}}$, если $u_{i}=0$, либо $T_{i}=L_{i}$, если $u_{i}=1$. Заметим, что любой полилинейный одночлен от $x_{1}, \ldots, x_{n}$ в $M\{X\}$ однозначно записывается в виде

$$
\left(x_{i} x_{j}\right) u\left(x_{i_{1}}, \ldots, x_{i_{n-2}}\right)
$$


где $u$ - двоичное слово длины $n-2$, а $\left\{i_{1}, \ldots, i_{n-2}\right\}=\{1, \ldots, n\} \backslash\{i, j\}$. Однозначность вытекает из следующей леммы.

Лемма 1 Элеменъ вида (5) линейно независимы.

Доказательство. Для бесконечного двоичного слова $w$ наряду с алгеброй $A(w)$ рассмотрим алгебру $C(w)$ с базисом $\left\{c_{0}, c_{1}, \ldots, c_{m}, \cdots\right\}$ и таблицей умножения

$$
c_{k}=\left\{\begin{array}{lll}
c_{k} c_{k-1}, & \text { если } & w_{k}=1, \\
c_{k-1} c_{k}, & \text { если } & w_{k}=0 .
\end{array}\right.
$$

для всех $k \geq 1$. Все остальные произведения базисных элементов считаем равными нулю. Зафиксируем элемент $z$ вида (5) и рассмотрим слово $w$, у которого начальное подслово длины $n-1$ равно $1 u$. Тогда подстановка $\varphi$, для которой

$$
\varphi\left(x_{i}\right)=c_{1}, \varphi\left(x_{j}\right)=c_{0}, \varphi\left(x_{i_{1}}\right)=c_{2}, \ldots, \varphi\left(x_{i_{n-2}}\right)=c_{n-1},
$$

дает нам ненулевое значение на $z$ :

$$
\varphi(z)=\varphi\left(\left(x_{i} x_{j}\right) u\left(x_{i_{1}}, \ldots, x_{i_{n-2}}\right)\right)=c_{n-1},
$$

тогда как

$$
\varphi\left(\left(x_{k} x_{l}\right) u^{\prime}\left(x_{t_{1}}, \ldots, x_{t_{n-2}}\right)\right)=0,
$$

если $\left(k, l, t_{1}, \ldots, t_{n-2}\right) \neq\left(i, j, i_{1}, \ldots, i_{n-2}\right)$, либо $u \neq u^{\prime}$.

Подслово $u$ бесконечного слова $w$ будем называть собственным, если хотя бы одно из его вхождений в $w$ начинается с $k$-й позиции, $k \geq 3$.

Лемма 2 Полилинейный одночлен $\left(y_{1} y_{2}\right) u\left(x_{1}, \ldots, x_{m}\right)$ не является тождеством алгебры $A(w)$ тогда и только тогда, когда и - собственное подслово в $w$.

Доказательство. Если $u=w_{i} \ldots w_{i+m-1}$ и $i \geq 3$, то

$$
b_{i-2} T_{a} u(a, \ldots, a)=b_{i+m-1} \neq 0,
$$

где $T_{a}=R_{a}$ при $w_{i-1}=0$, либо $T_{a}=L_{a}$ при $w_{i-1}=1$. Еси же $u$ не является собственным подсловом в $w$, то любая подстановка базисных элементов $A(w)$ вместо $y_{1}, y_{2}, x_{1}, \ldots, x_{m}$ дает нулевое значение.

Лемма 3 Для любого двоичного слова и и для любой подстановки $\sigma \in S_{m}$ в $A(w)$ выполняется тождество

$$
\left(y_{1} y_{2}\right) u\left(x_{\sigma(1)}, \ldots, x_{\sigma(m)}\right)-\left(y_{1} y_{2}\right) u\left(x_{1}, \ldots, x_{m}\right) \equiv 0 .
$$

Доказательство. Если $u$ не является собственным подсловом в $w$, то оба одночлена в (11) тождественно равны нулю в $A(w)$ согласно лемме 2. Если же $u-$ собственное подслово в $w$, то каждый из одночленов в (11) может принимать ненулевое значение только при $x_{1}=\cdots=x_{m}=a$.

Разделим собственные подслова в $w$ на две категории. Подслово $u$ называется подсловом 1-го типа, если оно встречается в $w$ только после нуля, либо только после единицы. Если же $u$ встречается в $w$ и после 0 и после 1 , то назовем его подсловом 2-го типа. 
Лемма 4 Пусть и - подслово 1-го типа в $w$. Если и следует всегда после нуля, то в $A(w)$ выполняются тождества

$$
\left(x_{i} x_{j}\right) u\left(x_{i_{1}}, \ldots, x_{i_{n-2}}\right) \equiv\left(x_{i} x_{1}\right) u\left(x_{2}, \ldots, x_{i-1}, x_{i+1}, \ldots, x_{n}\right),
$$

при $i>1 u\left\{i_{1}, \ldots, i_{n-2}\right\}=\{1, \ldots, n\} \backslash\{i, j\}$, а также

$$
\left(x_{1} x_{i}\right) u\left(x_{i_{1}}, \ldots, x_{i_{n-2}}\right) \equiv\left(x_{1} x_{2}\right) u\left(x_{3}, \ldots, x_{n}\right),
$$

где $\left\{i_{1}, \ldots, i_{n-2}\right\}=\{2, \ldots, n\} \backslash\{i\}$. Если и следует всегда после единицы, то в $A(w)$ выполняются тождества

$$
\left(x_{j} x_{i}\right) u\left(x_{i_{1}}, \ldots, x_{i_{n-2}}\right) \equiv\left(x_{1} x_{i}\right) u\left(x_{2}, \ldots, x_{i-1}, x_{i+1}, \ldots, x_{n}\right),
$$

при $i>1 u\left\{i_{1}, \ldots, i_{n-2}\right\}=\{1, \ldots, n\} \backslash\{i, j\}$, а тажже

$$
\left(x_{i} x_{1}\right) u\left(x_{i_{1}}, \ldots, x_{i_{n-2}}\right) \equiv\left(x_{2} x_{1}\right) u\left(x_{3}, \ldots, x_{n}\right),
$$

где $\left\{i_{1}, \ldots, i_{n-2}\right\}=\{2, \ldots, n\} \backslash\{i\}$. При этом элементы из правой части (12), $2 \leq i \leq n$, и элелент из правой части (13) линейно независимы. Такая же независимость выполняетя для правых частей (14), (15).

Доказательство. Докажем соотношение (12). Пусть $\varphi: X \rightarrow A(w)-$ подстановка базисных элементов $A(w)$ вместо порождающих. В силу тождества (4) и левая и правая части (12) переходят в ноль, если хотя бы два порождающих переходят в $\left\{b_{0}, b_{1}, \ldots\right\}$. Если $\varphi\left(x_{i}\right)=a$, то $\varphi\left(x_{i} x_{j}\right)=0$ для всех $j \neq i$, т.к. слову $u$ всегда предшествует ноль в $w$. Значение $\varphi$ на правой и левой частях (12) может быть ненулевым только если $\varphi\left(x_{i}\right)=b_{k}$ и $\varphi\left(x_{s}\right)=a$ для всех остальных $s$. Но при этом обе части (12) принимают значение $b_{k+n-1}$, что доказывает тождество (12). Соотношения (13), (14), (15) доказываются аналогично.

Покажем, что одночлены $f_{1}=\left(x_{1} x_{2}\right) u\left(x_{3}, \ldots, x_{n}\right)$ и

$$
f_{i}=\left(x_{i} x_{1}\right) u\left(x_{2}, \ldots, x_{i-1}, x_{i+1}, \ldots, x_{n}\right), 2 \leq i \leq n,
$$

линейно независимы по модулю идеала тождеств алгебры $A(w)$, если $u$ всегда следует за нулем в $w$. Предположим, что $f=\lambda_{1} f_{1}+\cdots+\lambda_{n} f_{n} \equiv 0-$ тождество. Пусть, например, $\lambda_{1} \neq 0$. Тогда для любого вхождения $u$ в $w$ существует такое $k \geq 0$, что подстановка

$$
\varphi\left(x_{1}\right)=b_{k}, \varphi\left(x_{2}\right)=\cdots=\varphi\left(x_{n}\right)=a
$$

дает значения

$$
\varphi\left(f_{1}\right)=b_{k+n-1}, \varphi\left(f_{2}\right)=\cdots=\varphi\left(f_{n}\right)=0,
$$

а это противоречит предположению что $f$ - тождество нашей алгебры. Аналогично, для любого $i>1$ можно найти подстановку $\varphi$, для которой $\varphi\left(f_{i}\right) \neq 0$, тогда как $\varphi\left(f_{j}\right)=0$ для всех $j \neq i$. Независимость правых частей (14) и (15) доказывается аналогично. 
Лемма 5 Пусть и - подслово 2-го типа в $w$. Тогда линейная оболочка одночленов

$f_{i j}=\left(x_{i} x_{j}\right) u\left(x_{l_{1}}, \ldots, x_{l_{n-2}}\right), i \neq j, l_{1}<\cdots<l_{n-2},\left\{l_{1}, \ldots, l_{n-2}\right\}=\{1, \ldots, n\} \backslash\{i, j\}$

в $M\{X\}$ по модулю тождеств алгебри $A(w)$ имеет размерность $r_{n}$, где $r_{n}-$ ранг системы $2 n$ уравнений на $n^{2}-n$ неизвестных $z_{i j}, 1 \leq i \neq j \leq n$,

$$
\sum_{i} z_{i j}=0, \quad 1 \leq j \leq n, \quad \sum_{j} z_{i j}=0, \quad 1 \leq i \leq n .
$$

Доказательство. Покажем, что линейная комбинация $f=\sum_{i, j} \lambda_{i j} f_{i j}-$ тождество $A(w)$ тогда и только тогда, когда набор коэффициентов $\left\{\lambda_{i j}\right\}$ является решением системы (20). Пусть сначала одно из равенств не выполняется, например, $\lambda=\lambda_{i 1}+\cdots+\lambda_{i n} \neq 0$. По определению $u$ в $w$ найдется начальное подслово вида $w_{1} \ldots w_{k+1} u$, в котором $w_{k+1}=0$, а $w_{k+2} \ldots w_{k+n-1}=u$. Обозначим $w_{1} \ldots w_{k}$ через $v$. Тогда по определению умножения в $A(w)$ имеем:

$$
b_{0} v(a, \ldots, a) a u(a, \ldots, a)=b_{k+n-1} .
$$

Рассмотрим подстановку $\varphi: \varphi\left(x_{i}\right)=b_{k}, \varphi\left(x_{t}\right)=a$ для всех $t \neq i$. Тогда

$$
\varphi\left(\left(x_{i} x_{j}\right) u\left(x_{l_{1}}, \ldots, x_{l_{n-2}}\right)\right)=b_{k+n-1}
$$

для любого $j \neq i$, а $\varphi(f)=\lambda b_{k+n-1} \neq 0$. Аналогично, $\lambda_{1 j} f_{1 j}+\cdots+\lambda_{n j} f_{n j}$ не является тождеством, если $\lambda_{1 j}+\cdots+\lambda_{n j} \neq 0$.

Теперь покажем, что $f \in \operatorname{Id}(\mathrm{A}(\mathrm{w}))$, если $\left\{\lambda_{i j}\right\}$ - решение системы (20). Значения всех одночленов $f_{i j}$ равны нулю при любой подстановке $\varphi$, у которой среди $\varphi\left(x_{1}\right), \ldots, \varphi\left(x_{n}\right)$ нет ни одного базиисного вектора $b_{k}$, либо не менее двух $b_{r}, b_{m}$. Предположим, что $\varphi\left(x_{i}\right)=b_{k}, \varphi\left(x_{j}\right)=a$ для всех $i \neq j$. Пусть снова $u=w_{k+2} \cdots w_{k+n-1}$. Если $w_{k+1}=0$ в слове $w$, то $b_{k} a=b_{k+1}, a b_{k}=0$. Поэтому

$$
\varphi\left(x_{i} x_{1}\right)=\ldots=\varphi\left(x_{i} x_{n}\right)=b_{k} a=b_{k+1}
$$

и $\varphi\left(f_{i j}\right)=b_{k+n-1}$ для всех $j \neq i$. В то же время $\varphi\left(f_{r t}\right)=0$ при $r \neq i$. Следовательно,

$$
\varphi(f)=\left(\sum_{j} \lambda_{i j}\right) b_{k+n-1}=0 .
$$

Аналогчно, если $w_{k+1}=1$, то

$$
\varphi\left(x_{1} x_{i}\right)=\ldots=\varphi\left(x_{n} x_{i}\right)=a b_{k}=b_{k+1}
$$

и

$$
\varphi(f)=\left(\sum_{j} \lambda_{j i}\right) b_{k+n-1}=0 .
$$

Пространство, порожденное всеми $f_{i j}$ из (19) в $M\{X\}$, имеет размерность $n^{2}-n$. Их линейные комбинации, являющиеся тождествами $A(w)$, образуют пространство размерности $n^{2}-n-r_{n}$, следовательно, коразмерность его пересечения с $\operatorname{Id}(\mathrm{A}(\mathrm{w}))$ и есть $r_{n}$. 
Замечание 1 Несложным упражнением из линейной алгебры является доказательство того, что $r_{n}=2 n-1$.

Теорема 1 Для алгебры $A(w)$ n-я коразмерность при $n \geq 3$ равна

$$
c_{n}(A(w))=k_{n-2}^{(1)} n+k_{n-2}^{(2)}(2 n-1),
$$

где $k_{m}^{(1)}, k_{m}^{(2)}$ - количество подслов 1-го и 2-го типов длины $m$ в ш. В частности,

$$
\operatorname{Comp}_{w *}(n-2) \leq c_{n}(A(w)) \leq 2 \operatorname{Comp}_{w *}(n-2)
$$

əде $w *=w_{3} w_{4} \ldots$

Доказательство. Обозначим через $P_{n, u}$ линейную оболочку в $M\{X\}$ всех одночленов (5) для любого двоичного слова $u$ длины $n-2$. Тогда

$$
P_{n}(M\{X\})=\bigoplus_{|u|=n-2} P_{n, u}
$$

Из леммы 2 следует, что по модулю идеала $\operatorname{Id}(\mathrm{A}(\mathrm{w}))$ пространство $P_{n}(M\{X\})$ содержит лишь те слагаемые из (29), у которых $u$ - собственные подслова в $w$. Пусть $u_{1}, \ldots, u_{t}$ - все собственные подслова длины $n-2$, и $f_{1} \in P_{n, u_{1}}, \ldots, f_{t} \in$ $P_{n, u_{t}}$. Нетрудно заметить, что если $f_{1}+\cdots+f_{t}$ - тождество $A(w)$, то и все $f_{1}, \ldots, f_{t}$ - тоже тождества. Теперь соотношение $(27)$ следует из лемм $3,4,5$, а (27) следует из очевидного соотношения $k_{n-2}^{(1)}+k_{n-2}^{(2)}=\operatorname{Comp}_{w *}(n-2)$.

Теорема 1 позволяет существенно расширить класс алгебр с промежуточным ростом коразмерностей. Например, в работе [3] для любой функции $\varphi: \mathbb{R}^{+} \rightarrow$ $\mathbb{R}^{+}$, такой, что

(i) $\varphi(t)>>\log t$

(ii) $\varphi(t)$ диффернцируема на $(0, \infty)$;

(iii) $\varphi^{\prime}(t)<<t^{-\beta}$ для некоторой константы $\beta>0$;

(iv) $\varphi^{\prime}-$ убывающая функция

существует двоичное слово $u$, для которого $\log C o m p_{u}(n) \sim \varphi(n)$. Здесь соотношение $f(t)<<g(t)$ означает, что

$$
\lim _{n \rightarrow \infty}(f(t) / g(t))=0,
$$

а под $\log$ подразумевается двоичный логарифм. С учетом теоремы 1 получаем следующую серию примеров.

Теорема 2 Для любой функции $\varphi(t)$, удовлетворяющей условиям (i) - (iv), существует алгебра $A$, для которой $c_{n}(A) \sim 2^{\varphi(n)}$. 
Новый класс функций промежуточного роста, реализуемых как рост коразмерностей, включает в себя, например, все функции $a^{\sqrt{n}}, a>1$. Более экзотический пример, приведенный в [3], соответствует функции

$$
\varphi(t)=(t+10)^{\frac{1}{2}+\frac{1}{4} \cos (\ln \ln (t+1))}
$$

которая очень медленно осциллирует между $n^{1 / 4}$ и $n^{3 / 4}$.

Опираясь на другие результаты теории формальных языков можно построить примеры алгебр и с более резкими колебаниями функции коразмерностей (см. [1, теорема 9]).

Теорема 3 Существует алгебра $A$, для которой можно выбрать возрастаюшую последовательность $n_{k}, k=1,2, \ldots$, так, что

(a) $c_{n_{k}}(A)<n_{k}+\ln \ln n_{k}$, если $k$ нечетно,

(b) $c_{n_{k}}>2^{\frac{n_{k}}{\ln \ln n_{k}}}$, если $k$ четно.

Теорема 1 позволяет построить пример алгебры, для которой существуют 1 й и 2-й пределы (2), но отсутсвует 3-й предел, т.е. опровергает гипотезу Регева в 3-м приближении. Для этого рассмотрим язык $E_{0}$, состоящий из всех слов в двухбуквенном алфивите $\{a, b\}$, не содержащих подслов $a^{2}, b^{4}, a b^{2} a$.

В работе [13] вычислена комбинаторная сложность языка $E_{0}$ :

$$
\operatorname{Comp}_{E_{0}}=\left\{\begin{array}{l}
2 F_{k+2}, \quad \text { если } n=2 k, \\
F_{k+4}, \quad \text { если } n=2 k+1,
\end{array}\right.
$$

где $F_{m}-m$-е число Фибоначчи. Построим слово $w$, у которого язык всех подслов совпадает с $E_{0}$. Для этого выпишем последовательно все слова из $E_{0}$ длины 1 , затем длины 2, и т.д. Кроме того, слова с началом $b a$ повторим дважды.

Далее, чтобы избежать появления запрещенных подслов, вставим при необходимости между соседними словами промежуточные слова длины не более 3-х. Например, между $a$ и $b^{2} a$ можно вставить $b$, а между $a b$ и $b a-a$ или $a b^{2}$. Более того, так как слова с префиксом $b a$ встречаются дважды, можно сделать дополнительные вставки таким образом, чтобы в одном случае такому слову $v$ предшествовала буква $a$, а в другом $b$.

Это возможно, поскольку между $a$ и $b a$ можно вставить и $b a$ и $b a b^{2}$, между $a b$ и $b a-a$ и $a b^{2}$, между $a b^{2}$ и $b a-$ пустое слово или $b a$, а между $a b^{3}$ и $b a-a$ или $a b^{2}$. Это позволяет построить слово $w$, язык которого совпадает с $E_{0}$ и все слова с началом $b a$ относятся к подсловам 2-го типа. Слова с началом $a, b^{2} a$ и $b^{3} a$ не могут быть подсловами 2-го типа, т.е. имеют тип 1.

Заметим, что $w *=w_{3} w_{4} \ldots$ по построению содержит все слова из $E_{0}$, поэтому чтобы применить теорему 1 достаточно подсчитать количесво подслов 1-го и 2-го типов в самом слове $w$.

Обозначим число подслов длины $k$ в $w$, начинающихся с $a$ через $\alpha_{k}$. Тогда любое такое подслово имеет вид $a b a v$, либо $a b^{3} a u$. Поэтому $\alpha_{k}=\alpha_{k-2}+\alpha_{k-4}$ при $k \geq 5$. Учитывая, что $\alpha_{1}, \alpha_{2}, \alpha_{3}, \alpha_{4}=1,1,2,2$, мы видим, что $\alpha_{2 k}=\alpha_{2 k-1}=F_{k+1}$ для всех $k \geq 1$. 
Обозначим через $\beta_{m}, \gamma_{m}, \delta_{m}$ количество подслов длины $m$ в $w$ с началом $b a, b^{2} a, b^{3} a$ соответственно. Тогда для четных индексов

$$
\beta_{2 k}=\alpha_{2 k-1}=F_{k+1}, \quad \gamma_{2 k}=\alpha_{2 k-2}=F_{k}, \quad \delta_{2 k}=\alpha_{2 k-3}=F_{k},
$$

а для нечетных

$$
\beta_{2 k+1}=\alpha_{2 k}=F_{k+1}, \quad \gamma_{2 k+1}=\alpha_{2 k-1}=F_{k+1}, \quad \delta_{2 k+1}=\alpha_{2 k-3}=F_{k} .
$$

Отсюда

$$
k_{n-2}^{(1)}=\left\{\begin{array}{lll}
F_{k-1}+F_{k+1} & \text { при } & n=2 k \\
F_{k-1}+F_{k+2} & \text { при } & n=2 k+1,
\end{array}\right.
$$

a

$$
k_{n-2}^{(2)}=\beta_{n-2}=F_{k}
$$

и при $n=2 k$ и при $n=2 k+1$. Из $(35),(36)$ и теоремы 1 следует что для слова $w$ и построенной по нему алгебры $A(w)$ мы имеем

$$
c_{n}(A(w))=n\left(F_{t-1}+F_{t+1}\right)+(2 n-1) F_{t}
$$

при $n=2 t$ и

$$
c_{n}(A(w))=n\left(F_{t-1}+F_{t+2}\right)+(2 n-1) F_{t}
$$

при $n=2 t+1$. Поскольку

$$
F_{t}=\frac{\varphi^{t}+(-\varphi)^{-t}}{2 \varphi-1} \sim \frac{\varphi^{t}}{1+\sqrt{5}}, \quad \text { где } \quad \varphi=\frac{1+\sqrt{5}}{2}
$$

а коэффициент при $n$ у $c_{n}(A(w))$ равен $F_{t-1}+2 F_{t}+F_{t+1}$ при $n=2 t$ и $F_{t-1}+$ $2 F_{t}+F_{t+2}$ при $n=2 t+1$, то

$$
\lim _{n \rightarrow \infty} \sqrt[n]{c_{n}(A(w))}=\sqrt{\varphi}, \quad \lim _{n \rightarrow \infty} \log _{n} \frac{c_{n}(A(w))}{\sqrt{\varphi}^{n}}=1 .
$$

В то же время 3-го предела в (2) не существует, поскольку

$$
\lim _{n=2 t \rightarrow \infty} \log _{n} \frac{c_{n}(A(w))}{n \sqrt{\varphi}^{n}}=\frac{\varphi^{2}+\varphi+2}{\varphi(2 \varphi-1)}, \lim _{n=2 t+1 \rightarrow \infty} \log _{n} \frac{c_{n}(A(w))}{n \sqrt{\varphi}^{n}}=\frac{\varphi^{3}+\varphi+2}{\varphi(2 \varphi-1)} .
$$

Основные результаты статьи анонсированы в [15].

Работа первого автора поддержана Российским научным фондом, грант No 16-11-10013. Работа второго автора поддержана Словенским исследовательским агенством, грант No P1-0292. 


\section{Список литературы}

[1] J. Balogh, B. Bollobas, Hereditary properties of words, Theor. Inform. Appl. 39 (2005), no. 1, 49-65.

[2] A. Berele, Properties of hook Schur functions with applications to p.i. algebras, Adv. Appl. Math. 41 (2008), no. 1, 52-75.

[3] J. Cassaigne, Constructing infinite words of intermediate complexity, Lect. Notes Comp. Sci. 2450 (2003), 173-184.

[4] A. Giambruno, S. Mishchenko, M. Zaicev, Algebras with intermediate growth of the codimensions, Adv. Appl. Math. 37 (2006), no. 3, 360-377.

[5] A. Giambruno, S. Mishchenko, M. Zaicev, Codimensions of algebras and growth functions, Adv. Math. 217 (2008), no 3, 1027-1052.

[6] A. Giambruno, M. Zaicev. On codimension growth of finitely generated associative algebras, Adv. Math. 140 (1998), no. 2, 145-155.

[7] A. Giambruno, M.V. Zaicev, Exponential codimension growth of PI algebras: an exact estimate, Adv. Math. 142 (1999), 221-243.

[8] A. Giambruno, M. Zaicev, Polynomial Identities and Asymptotic Methods, Mathematical Surveys and Monographs. 122, Amer. Math. Soc., Providence, RI, 2005.

[9] A. Giambruno, M. Zaicev, Growth of polynomial identities: is the sequence of codimensions eventually non-decreasing? Bull. Lond. Math. Soc. 46 (2014), no. 4, 771-778.

[10] A. Giambruno, M. Zaicev, Anomalies on codimension growth of algebras, Forum Math. 28 (2016), no. 4. 649-656.

[11] С.П. Мищенко, Н.П. Панов. Слова Штурма и несчетное множество почти нильпотентных многообразий квадратичного роста, Вестник Моск. Ун-та, Cер.1, Матем., Механ, 2017, No. 6, С. 55-59.

[12] A. Regev, Existence of identities in $A \otimes B$, Israel J. Math. 11 (1972), 131-152.

[13] А. М. Шур, О вычислении параметров и типов поведения комбинаторной сложности регулярных языков, Тр. ИММ УрО РАН, 2010, том 16, номер $2,270-287$.

[14] M. Zaicev, On existence of PI-exponents of codimension growth, Electr. Researh Announ. Math. Sci. 21 (2014), 113-119.

[15] М.В. Зайцев, Д.Д. Реповш, Тождества в алгебрах и комбинаторные свойства двоичных слов. Доклады академии наук, 2019, том 489, номер 5, 449451. 